\title{
Static Stability of Double-Spiral Mobile Robot over Rough Terrain
}

\author{
Naohiko Hanajima ${ }^{1}$ Taiki Kaneko ${ }^{2}$ Hidekazu Kajiwara ${ }^{3}$ Yoshinori Fujihira ${ }^{1}$ \\ ${ }^{1}$ College of Design and Manufacturing Technology, Muroran Institute of Technology, Japan, \\ $\{$ hana, yfuji\}@mmm. muroran-it.ac.jp \\ ${ }^{2}$ Division of Production Systems Engineering, Muroran Institute of Technology, Japan \\ ${ }^{3}$ College of Information and Systems, Muroran Institute of Technology, Japan, \\ $\{$ kajiwara\} @mmm. muroran-it.ac.jp
}

\begin{abstract}
In this paper, we investigate static stability for a doublespiral mobile robot. It is a new locomotion mechanism suitable for the wetlands that suppresses damage to vegetation and does not sink in the mud. The robot walks on the spirals, which play the role of footholds for the mobile robot. To overcome rough terrain locomotion, we need to ensure the stability of the walking motion on the sloping ground. In this study, we applied normalized energy stability margin (NESM) to the double-spiral mobile robot in order to investigate its static stability over rough terrain. The procedure to derived the NESM value were shown from the point of view of the vector calculation. In the numerical case study, we drew NESM maps to investigate the static stabilities when the inclination of the slope varied or the pose and orientation of the robot changed. We adopted a moment in the swing phase where the stability of the robot's balance was easily lost. We found that the robot has sensitive directions in terms of stability. Planning the route and motion of the robot in the rough terrain could help maintain its stability.
\end{abstract}

Keywords: wetland, field survey, mobile robot, slope, balance

\section{Introduction}

The problem regarding the reduction of wetlands areas has come to occupy an important position in environmental conservation (Nakamura et al., 2004; Fujita et al., 2009). To investigate the mechanisms of wetlands degradation trends, much effort is put into field surveys (Musgrave and Binley, 2011; Riddell et al., 2010). The survey area where we focus is deeply covered with alder forests or Sasa (veitchii) and the ground surface is formed by a thick pile of withered Sasa stems on the muddy soil and water. It is difficult for researchers to walk around huge areas in the wetlands with large quantities of survey tools. Therefore, a technical support system for field surveys in the wetlands is required.

Recently a double-spiral mobile architecture has been proposed (Hanajima et al., 2009, 2016). It is a new locomotion mechanism suitable for the wetlands that minimizes damage to the vegetation and does not sink in the mud. It consists of two pairs of spirals and one quadruped mobile robot. Each pair of spirals plays the role of footholds for the mobile robot. By traveling at a higher distance from the ground over the spirals, the robot can avoid strong resistance from the dense and hard-stemmed plants. In addition, because the spiral is supported by several contact points on the ground and intermediates between the robot and the muddy ground, the robot never touches the ground and barely sinks.

The quadruped mobile robot has gantry-shaped legs for static walking on the spirals. Each of the legs is connected to a body platform with a rotational joint and two prismatic joints in perpendicular arrangement. Therefore, while all four legs maintain their position, the body platform can change its position and orientation in the horizontal plane while staying level. This provides the body platform with the capacity for arbitrary planar motion in its own plane.

The static walking motion of the double-spiral mobile robot on the flat ground has been performed successfully in numeric simulation. However, rough terrain is inherent in the survey area. We need to account for the stability of the walking motion on rough terrain.

Several stability criteria have been proposed in the field of multi-legged and multi-wheeled systems. For quadruped robots, stability margin was proposed in a very early stage (McGhee and Frank, 1968). It represents the distance between the projection of the center of gravity (COG) on the ground and the border of the supporting polygon. The tip-over stability margin, which is also called force-angle stability margin, is given by product of the magnitude of the net force acting on the COG and the minimum of the angles formed between the direction of the net force and the direction of the tip-over axis normal (Papadopoulos and Rey, 1996). Energy stability margin evaluates the minimum potential energy required to tumble the robot (Messuri and Klein, 1985). Normalized energy stability margin (NESM) is one of the improvements of it (Hirose et al., 2001). It is given by dividing the robot's weight into the energy stability margin for normalization; that is, it becomes the height difference of the COG. In this research, we deal with static stability in walking motion. We do not have to consider the forces other than the gravity acting on the robot. Therefore, we decided to employ NESM for the stability criteria, which 


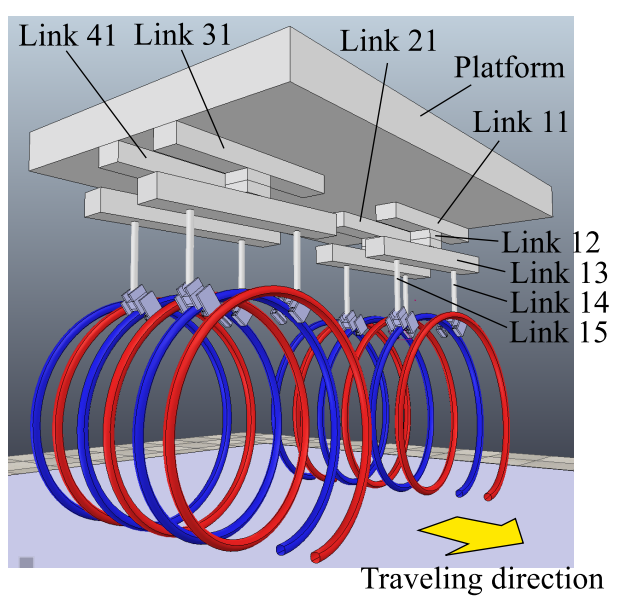

Figure 1. Whole image of the double-spiral robot and definition of links.

only consider the height of the COG of the robot (Kaneko and Hanajima, 2016).

For a quadruped robot, a swing phase is one of the least stable postures in the walking motion. A creep gait is a well-known static gait in which only one leg can be lifted at any given time (McGhee and Frank, 1968). For a quadruped, when one leg is lifted, the remaining three legs must support the weight of its body. The ground contact points of the three legs form a supporting polygon. Before the swing leg is lifted, the COG of the robot needs to be inside of the support polygon. At the same time, to enhance static stability, it is expected that the COG is located where its NESM is maximized.

In this study, we investigate the NESM values of the double-spiral mobile robot in the swing phase on several gradients of slope from the numerical case study. The knowledge obtained by the case study can be applied to the stable motion planning for the robot's static walking.

In the rest of this paper, first, we review the structure of the double-spiral mobile robot and its kinematics. Next, we introduce the NESM, followed by the numerical case study. Then, we show the results and discuss the potential of the stability of the robot.

\section{Structure and Kinematics of the Double-Spiral Mobile Robot}

\subsection{Structure}

Figure 1 shows the structure of the double-spiral mobile robot in the dynamics simulator. As mentioned in the introduction, it consists of two pairs of spirals and one mobile robot. The mobile robot possesses four legs with a gantry-shaped mechanism. Links 11, 21, 31, and 41 move in the direction of travel with respect to the platform with prismatic joints $\mathrm{J} 11, \mathrm{~J} 21, \mathrm{~J} 31$, and $\mathrm{J} 41$, respectively. Note that the joints are embedded and invisible in Figure 1. We use 2 digits to specify the links and joints of the robot. The first digit denotes a leg number; Leg 1 represents front left; Leg 2, rear left; Leg 3, front right; and Leg 4, rear right.

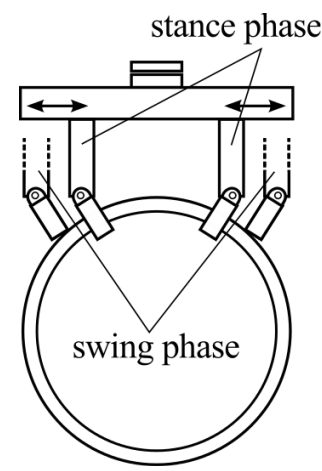

Figure 2. Stance phase and swing phase produced by motion of a gantry-shaped mechanism.

Hereafter, we only explain Leg 1 , because every leg has the same structure. Another prismatic joint, J12, is installed at Link 11, perpendicular to the traveling direction. It drives Link 12. The rotational joint, J13, rotates Link 13, which is a part of the legs. As a result, each leg has two prismatic joints orthogonal to each other and one rotational joint for 3 DOF motion in a plane.

As shown in Figure 1, the end link of each leg has a gantry-shaped mechanism. A pair of vertical links, Links 14 and 15, stands on the spiral in parallel. The distance between them is adjustable by the prismatic joints, J14 and J15. They are driven by one actuator, symmetrically. A gripper is mounted on the lower end of every vertical link. Each gripper requires special mechanisms to grip the rounded rim of the spiral stably so as not to slide down in the stance phase. Once the gripper holds the spiral, the leg needs to maintain its foot position even if the platform moves toward a different posture. In the creep gait, Links 14 and 15 take the opening motion in the swing phase and the closing motion in the stance phase, as shown in Figure 2. In the stance phase, the grippers must hold the spiral tightly.

Note that the working space of the grippers mounted on Link 14 and Link 15 forms the plane parallel to the body platform as well. When the position of the spiral and the working space of the grippers are given, their intersections indicate a pair of points to be gripped. Connecting the pair of points forms a line segment. By aligning Link 13 parallel to the line segment and making the distance between Links 14 and 15 equal to the length of the line segment, each gripper is located at the point to be gripped. In total, the leg mechanism should have enough DOF for the gripper to control a specific position. Since the four legs have the same properties, the position and posture angle of the body platform can be decided within the plane parallel to the grippers' working space independently of any gripper's position.

In total, the robot has enough DOF for its body to move in the desired direction. This function is also important to maintain the balance of the robot. To maintain its balance, the platform of the robot needs to be able to move arbitrarily in the horizontal plane as the NESM value be- 


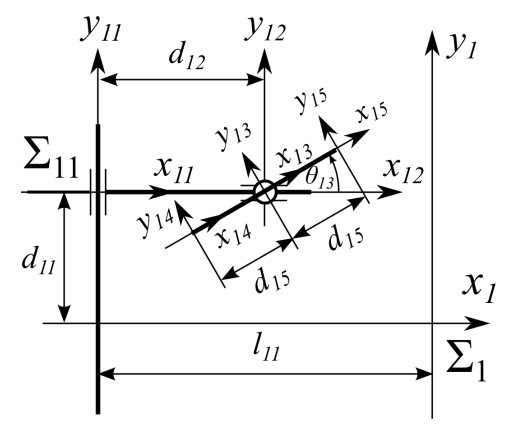

(a) top view

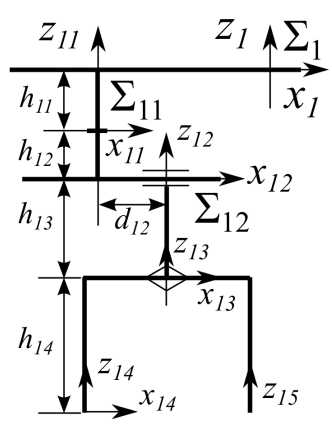

(b) rear view

Figure 3. The coordinate system and joints for one leg of the robot.

comes larger, even though the grippers continue to hold the same places on the spirals.

\subsection{Forward kinematics}

For the sake of mathematical consideration, we need to define a coordinate system at each link of the robot. Figure 3 shows the coordinate systems of the body platform and Leg 1 from the top view (a) and the rear view (b). We represent the coordinate system of the body as $\Sigma_{1}$ and each coordinate system of Link $\langle a\rangle$ as $\Sigma_{\langle a\rangle}$, respectively. The symbol $O_{\langle a\rangle}$ denotes the origin of $\Sigma_{\langle a\rangle}$, and $x_{\langle a\rangle}, y_{\langle a\rangle}$, and $z_{\langle a\rangle}$ denote the $x, y$, and $z$ axes of $\Sigma_{\langle a\rangle}$, respectively, according to the right-handed coordinate system. We define notation of a position vector of a point $p$ with respect to $\Sigma_{\langle a\rangle}$ as ${ }^{a} \boldsymbol{p}$.

Forward kinematics of a robot is usually represented by homogeneous transformation. The $3 \times 3$ block matrix, which consists of the first 3 rows and the first 3 columns of a homogeneous transformation matrix, represents a rotation matrix. The $3 \times 1$ vector, which consists of the first 3 rows in the fourth column of a homogeneous transformation matrix, represents a position vector. The position vector can be used to calculate the working space of a link.

With reference to Figure 3, the homogeneous transformation matrices between adjoining coordinate systems for Leg 1 are as follows.

$$
{ }^{1} H_{11}=\left[\begin{array}{cccc}
1 & 0 & 0 & -l_{11} \\
0 & 1 & 0 & d_{11} \\
0 & 0 & 1 & -h_{11} \\
0 & 0 & 0 & 1
\end{array}\right]
$$

$$
\begin{aligned}
& { }^{11} \boldsymbol{H}_{12}=\left[\begin{array}{cccc}
1 & 0 & 0 & d_{12} \\
0 & 1 & 0 & 0 \\
0 & 0 & 1 & -h_{12} \\
0 & 0 & 0 & 1
\end{array}\right] \\
& { }^{12} \boldsymbol{H}_{13}=\left[\begin{array}{cccc}
\cos \theta_{13} & -\sin \theta_{13} & 0 & 0 \\
\sin \theta_{13} & \cos \theta_{13} & 0 & 0 \\
0 & 0 & 1 & -h_{13} \\
0 & 0 & 0 & 1
\end{array}\right] \\
& { }^{13} \boldsymbol{H}_{14}=\left[\begin{array}{cccc}
1 & 0 & 0 & -d_{15} \\
0 & 1 & 0 & 0 \\
0 & 0 & 1 & -h_{14} \\
0 & 0 & 0 & 1
\end{array}\right] \\
& { }^{13} \boldsymbol{H}_{15}=\left[\begin{array}{cccc}
1 & 0 & 0 & d_{15} \\
0 & 1 & 0 & 0 \\
0 & 0 & 1 & -h_{14} \\
0 & 0 & 0 & 1
\end{array}\right]
\end{aligned}
$$

where ${ }^{a} \boldsymbol{H}_{b}$ represents a homogeneous transformation matrix from coordinate system $\langle a\rangle$ to coordinate system $\langle b\rangle$. The values of $h_{11}, h_{12}, h_{13}$, and $h_{14}$ are the offset distances in the $z_{1}$ axis direction between the $x_{1}$ axis, $x_{11}$ axis, $x_{12}$ axis, $x_{13}$ axis, and $x_{14}$ axis, respectively. The value of $l_{11}$ is the offset distance in the $x_{1}$ axis direction between the $y_{1}$ axis and $y_{11}$ axis. The variables of $d_{11}, d_{12}$, and $d_{15}$ are the joint displacements in the direction of the $y_{1}$ axis, $x_{11}$ axis, and $x_{13}$ axis, respectively. The variable of $\theta_{13}$ is the joint angle around the $z_{13}$ axis.

\subsection{Gripper positions}

The calculation of NESM requires specifying the positions of the grippers. The position vectors of grippers ${ }^{1} p_{14}$ and ${ }^{1} \boldsymbol{p}_{15}$ with respect to $\Sigma_{1}$ are represented as follows.

$$
\begin{aligned}
{ }^{1} \boldsymbol{p}_{14} & =\boldsymbol{I}_{3 \times 4}{ }^{1} \boldsymbol{H}_{11}{ }^{11} \boldsymbol{H}_{12}{ }^{12} \boldsymbol{H}_{13}{ }^{13} \boldsymbol{H}_{14} \boldsymbol{p}_{O} \\
& =\left[\begin{array}{c}
-l_{11}+d_{12}-d_{15} \cos \theta_{13} \\
d_{11}-d_{15} \sin \theta_{13} \\
-h_{11}-h_{12}-h_{13}-h_{14}
\end{array}\right] \\
{ }^{1} \boldsymbol{p}_{15} & =\boldsymbol{I}_{3 \times 4}{ }^{1} \boldsymbol{H}_{11}{ }^{11} \boldsymbol{H}_{12}{ }^{12} \boldsymbol{H}_{13}{ }^{13} \boldsymbol{H}_{15} \boldsymbol{p}_{O} \\
& =\left[\begin{array}{c}
-l_{11}+d_{12}+d_{15} \cos \theta_{13} \\
d_{11}+d_{15} \sin \theta_{13} \\
-h_{11}-h_{12}-h_{13}-h_{14}
\end{array}\right]
\end{aligned}
$$

where

$$
\boldsymbol{I}_{3 \times 4}=\left[\begin{array}{cccc}
1 & 0 & 0 & 0 \\
0 & 1 & 0 & 0 \\
0 & 0 & 1 & 0
\end{array}\right], \quad \boldsymbol{p}_{O}=\left[\begin{array}{l}
0 \\
0 \\
0 \\
1
\end{array}\right]
$$

The position vectors of the grippers for the other legs are defined in the same manner.

\subsection{COG}

(1) The calculation of NESM also requires the COG of the whole robot. The robot body is divided into three portions from the aspect of the COG; the body platform, the first 


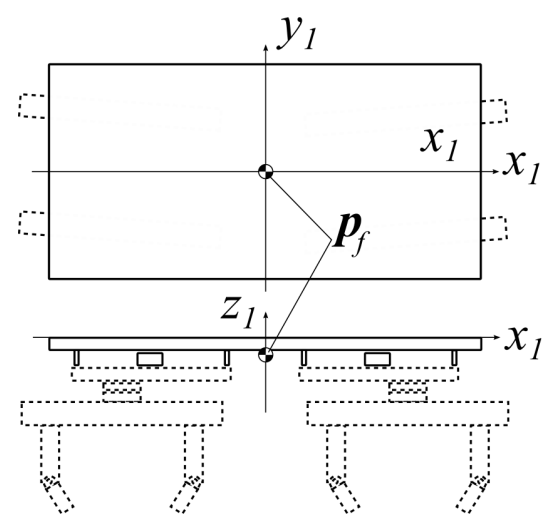

Figure 4. COG of the platform.

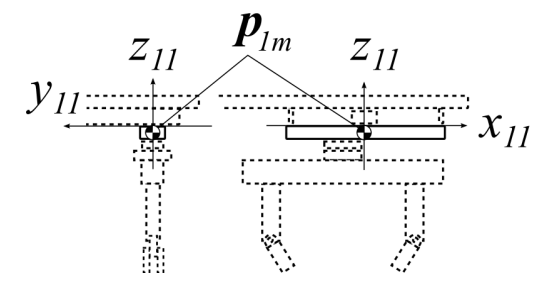

Figure 5. COG of Link 11.

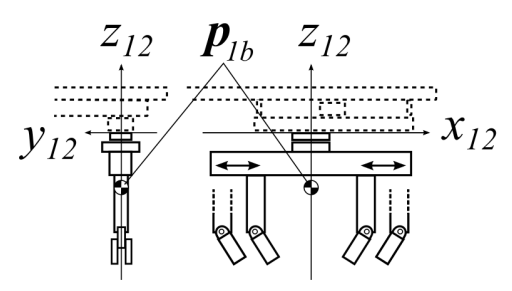

Figure 6. COG of Link 12.

links for the four legs, and the four gantry-shaped legs. We define the position vectors for each portion as $\boldsymbol{p}_{f}, \boldsymbol{p}_{n m}$, and $\boldsymbol{p}_{n b}$ as shown in Figure 4, Figure 5, and Figure 6, respectively, where $n$ denotes the leg number. The position vector of the total COG of the whole robot, $\boldsymbol{p}_{c}$, is obtained by the following equation.

$$
\boldsymbol{p}_{c}=\frac{m_{f} \boldsymbol{p}_{f}+\sum_{n=1}^{4}\left(m_{n m} \boldsymbol{p}_{n m}+m_{n b} \boldsymbol{p}_{n b}\right)}{m_{f}+\sum_{n=1}^{4}\left(m_{n m}+m_{n b}\right)}
$$

where $m_{f}, m_{n m}$, and $m_{n b}$ denote the mass of the three portions: the body platform, the first links for the four legs, and the four gantry-shaped legs, respectively.

\section{NESM}

In this section, we apply the NESM to the double-spiral mobile robot on a slope and show the calculation method.

First, we define a reference coordinate frame $\Sigma_{R}$ in the field. It is supposed that the double-spiral mobile robot is located on the slope as shown in Figure 7. The axes $x_{R}$, $y_{R}$, and $z_{R}$ denote the principal axes of $\Sigma_{R}$. The positive direction of $z_{R}$ is upward to the vertical line. The plane consisting of $x_{R}$ and $y_{R}$ is set arbitrarily.

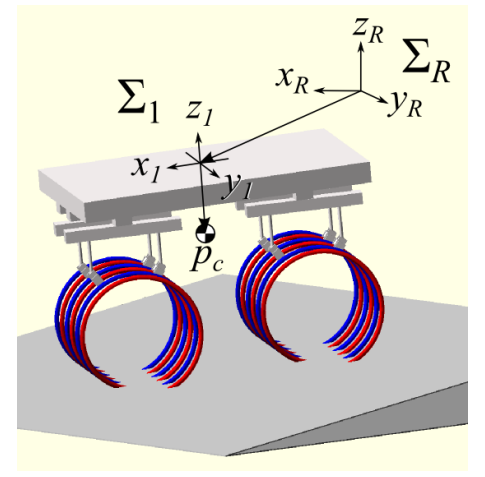

Figure 7. Reference frame and relationship of frames.

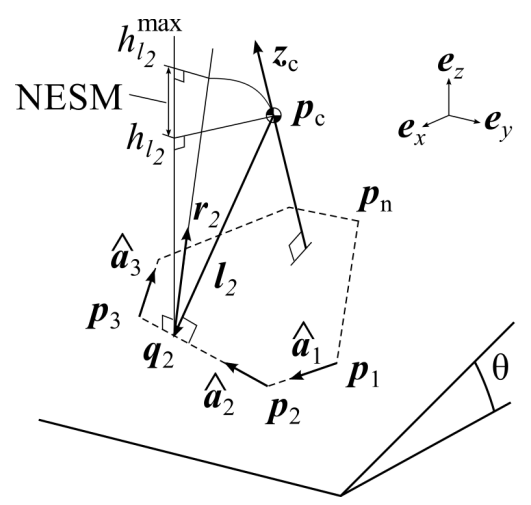

Figure 8. Illustration of normalized energy stability margin.

To derive NESM, the height information in the reference coordinate frame is mandatory. We define the homogenous transfer matrix ${ }^{R} \boldsymbol{H}_{1}$ from $\Sigma_{R}$ to $\Sigma_{1}$, which represents the position and orientation of the whole robot. We can obtain the position vectors with respect to $\Sigma_{R}$ by premultiplying the position vector with respect to $\Sigma_{1}$ by ${ }^{R} \boldsymbol{H}_{1}$. After the position vectors of the COG and the grippers of the four legs are obtained, their coordinates are converted to those with respect to $\Sigma_{R}$ using ${ }^{R} \boldsymbol{H}_{1}$. Hereafter, we use the notation $\boldsymbol{p}_{c}$ for the position vector with respect to $\Sigma_{R}$.

In the stance phase, all eight grippers hold the spirals. In the swing phase, one pair of grippers releases one of the spirals. Before the moment of release, the body platform needs to be located at the position where its NESM in the swing phase is maximized. The positions of the six grippers that will hold the spiral in the swing phase form a polygon. However, the polygon does not always form a convex polygon. By applying a quickhull algorithm (Barber et al., 1996) to the positions of the six grippers, we can obtain the vertices of a convex polygon that forms a support polygon in the swing phase at the same time. We refer to the vertices of the support polygon as $p_{i}$, where $i$ is an index number.

Figure 8 illustrates NESM. Each $\boldsymbol{p}_{i}$ denotes a position vector relative to the vertex $p_{i}$. The support polygon is represented by broken lines. It is important that the index $i$ is ordered clockwise in the top view. When a tip over occurs, one edge of the polygon can be a rotation axis. A 


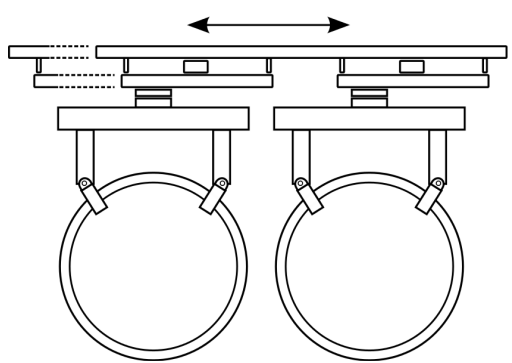

Figure 9. Motion of the platform: front view.

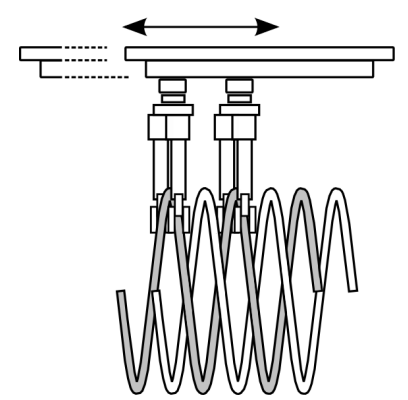

Figure 10. Motion of the platform: side view.

tip-over vector $\boldsymbol{a}_{i}$ is defined as the following equation so that it is aligned with the edge.

$$
\boldsymbol{a}_{i}=\boldsymbol{p}_{i+1}-\boldsymbol{p}_{i} \quad(i=1, \cdots, n)
$$

where $\boldsymbol{p}_{n+1} \equiv \boldsymbol{p}_{1}$. A tip-over unit vector $\hat{\boldsymbol{a}}_{i}$ has same direction as $\boldsymbol{a}_{i}$; that is, $\hat{\boldsymbol{a}}_{i}=\boldsymbol{a}_{i} /\left|\boldsymbol{a}_{i}\right|$. A vector $\boldsymbol{z}_{c}$ is normal to the support polygon passing through the COG of the whole robot. The position vector of the COG is shown as $\boldsymbol{p}_{c}$. A tip-over vector normal $\boldsymbol{l}_{i}$ is chosen so that it intersects the COG and the edge regarding $\boldsymbol{a}_{i}$. It is given by the following equation.

$$
\boldsymbol{l}_{i}=\left(\boldsymbol{I}-\hat{\boldsymbol{a}}_{i} \hat{\boldsymbol{a}}_{i}^{T}\right)\left(\boldsymbol{p}_{i+1}-\boldsymbol{p}_{c}\right)
$$

where $\boldsymbol{I}$ is the identity matrix. The position vector $\boldsymbol{q}_{i}$ is chosen where an intersection of $\boldsymbol{l}_{i}$ and $\boldsymbol{a}_{i}$. It is obtained by the following equation.

$$
\boldsymbol{q}_{i}=\boldsymbol{l}_{i}+\boldsymbol{p}_{c}
$$

Unit vectors $\boldsymbol{e}_{x}, \boldsymbol{e}_{y}$, and $\boldsymbol{e}_{z}$ are alined to the respective axes of the reference frame. The height of COG with respect to the reference frame, $h_{l_{i}}$, is equivalent to the $z$ axis component of $\boldsymbol{p}_{c}$. Therefore, it is obtained by the following equation.

$$
h_{l_{i}}=\boldsymbol{e}_{z}^{T} \boldsymbol{p}_{c}
$$

When the robot tumbles about the axis regarding $\boldsymbol{a}_{i}$, the locus of COG follows a circular path. The highest point of the locus is just above the axis regarding $\boldsymbol{a}_{i}$. The vector $\boldsymbol{r}_{i}$ is oriented from the position regarding $\boldsymbol{q}_{i}$ to the highest point. A vector given by a cross product of $\boldsymbol{e}_{z}$ and $\boldsymbol{a}_{i}$ is the normal vector of the plane including vector $\boldsymbol{r}_{i}$, which is perpendicular to $\boldsymbol{a}_{i}$. Therefore, the following relationship is satisfied.

$$
\boldsymbol{r}_{i}=\boldsymbol{a}_{i} \times\left(\boldsymbol{e}_{i} \times \boldsymbol{a}_{i}\right)
$$

The radius of the circle drawn by the COG is equal to $\left|\boldsymbol{l}_{i}\right|$. Then, the height of the highest point with respect to the reference frame, $h_{l_{i}}^{\max }$, is obtained by the following equation.

$$
h_{l_{i}}^{\max }=\boldsymbol{e}_{z}^{T}\left(\boldsymbol{q}_{i}+\left|\boldsymbol{l}_{i}\right| \hat{\boldsymbol{r}}_{i}\right)
$$

Finally, NESM for all edges of the support polygon is obtained by the following equation.

$$
\begin{aligned}
\mathrm{NESM} & =\min _{i}\left(h_{l_{i}}^{\max }-h_{l_{i}}\right) \sigma_{i} \\
& =\min _{i} \boldsymbol{e}_{z}^{T}\left(\boldsymbol{l}_{i}+\left|\boldsymbol{l}_{i}\right| \hat{\boldsymbol{r}}_{i}\right) \sigma_{i}
\end{aligned}
$$

where $\hat{\boldsymbol{r}}_{i}$ is a unit vector in the same manner as $\hat{\boldsymbol{a}}_{i}$, and $\sigma_{i}$ represents a sign of NESM, which is positive when the $\mathrm{COG}$ is in the stable side and negative otherwise. $\sigma_{i}$ is defined as the following formula.

$$
\sigma_{i}=\left(\hat{\boldsymbol{r}}_{i} \times \hat{\boldsymbol{l}}_{i}\right)^{T} \hat{\boldsymbol{a}}_{i}
$$

where $\hat{\boldsymbol{l}}_{i}$ is a unit vector in the same manner as $\hat{\boldsymbol{a}}_{i}$.

\section{Numerical Case Study}

In this section, we investigate the NESM values of the double-spiral mobile robot in the swing phase on several gradients of slope.

\subsection{Conditions and methods}

Table 1 shows the values used in the numerical case study, such as link parameters, platform sizes, weight, joint variables, and so on. The number $n$ in the subscript denotes the leg number.

The parameters $h_{p g}, h_{l n}$, and $h_{l g}$ represent positions of the COGs of the three portions. We suppose that the density of each portion is uniform. Therefore, we can assume that $\boldsymbol{p}_{f}$ and $\boldsymbol{p}_{n m}$ locate on the $z_{1}$ axis and $z_{n 1}$ axis, respectively, and that $\boldsymbol{p}_{n b}$ locates on the $z_{n 2}$ axis because Link $n 4$ and Link $n 5$ move symmetrically. As a result, we have the following:

$$
{ }^{1} \boldsymbol{p}_{f}=\left[\begin{array}{c}
0 \\
0 \\
h_{p g}
\end{array}\right],{ }^{n 1} \boldsymbol{p}_{n m}=\left[\begin{array}{c}
0 \\
0 \\
h_{l n}
\end{array}\right],{ }^{n 2} \boldsymbol{p}_{n b}=\left[\begin{array}{c}
0 \\
0 \\
h_{l g}
\end{array}\right] .
$$

In this numerical case study, we set the initial values of the joint parameters as shown in Table 1, such that the double-spiral mobile robot is in the swing phase -i.e., Leg 1 is a swing leg. After that, we move the platform to the position that is taken at each of twenty points at regular intervals within the full stroke of each joint in the $x_{1}$ and $y_{1}$ directions, as in Figure 9 and Figure 10, respectively. During the movement, the leg positions remain stationary. The ranges of the joint parameters are designated as strokes in Table 1. After calculating the values of NESM 
Table 1. The values used in the numerical case study. $n$ in the subscript denotes the leg number.

\begin{tabular}{lrrrr}
\hline Item & Value & & Item & Value \\
\cline { 1 - 2 } \cline { 5 - 5 }$l_{n 1}$ & $0.5[\mathrm{~m}]$ & & width of platform & $1.0[\mathrm{~m}]$ \\
$h_{n 1}$ & $0.1[\mathrm{~m}]$ & & depth of platform & $0.5[\mathrm{~m}]$ \\
$h_{n 2}$ & $0.05[\mathrm{~m}]$ & & height of platform & $0.1[\mathrm{~m}]$ \\
$h_{n 3}$ & $0.1[\mathrm{~m}]$ & stroke of joint $1,2,4$ & $0.4[\mathrm{~m}]$ \\
$h_{n 4}$ & $0.5[\mathrm{~m}]$ & stroke of joint 3 & $0.4 \pi[\mathrm{rad}]$ \\
$h_{p g}$ & $0.1[\mathrm{~m}]$ & & $d_{11}, d_{31}$ & $0.2[\mathrm{~m}]$ \\
$h_{l n}$ & $0.025[\mathrm{~m}]$ & & $d_{21}, d_{41}$ & $-0.2[\mathrm{~m}]$ \\
$h_{l g}$ & $0.3[\mathrm{~m}]$ & & $d_{n 2}$ & $0.0[\mathrm{~m}]$ \\
$m_{f}$ & $100[\mathrm{~kg}]$ & $\theta_{n 3}$ & $\pi / 18[\mathrm{rad}]$ \\
$m_{n m}$ & $5[\mathrm{~kg}]$ & $d_{14}$ & $0.3[\mathrm{~m}]$ \\
$m_{n b}$ & $20[\mathrm{~kg}]$ & & $d_{24}, d_{34}, d_{44}$ & $0.2[\mathrm{~m}]$ \\
\hline
\end{tabular}

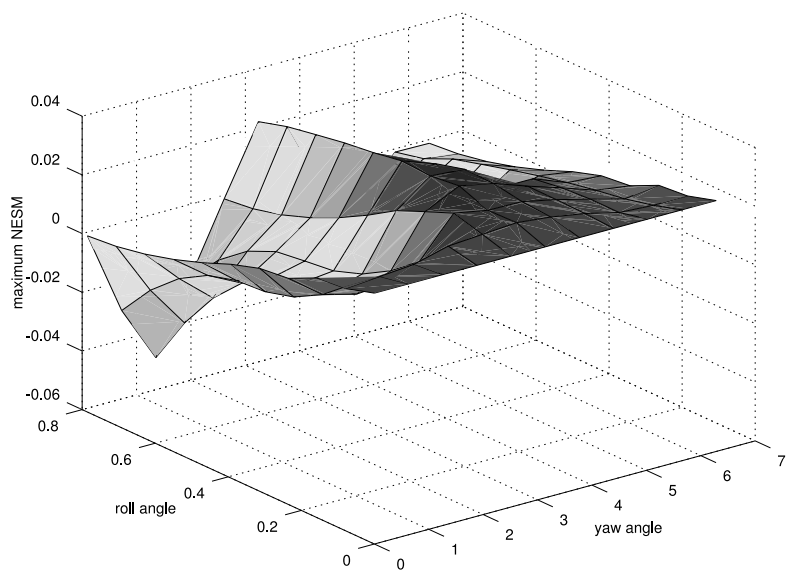

Figure 11. Maximum value map of NESM with respect to the yaw and roll angle of slope gradient.

at each position, the maximum value of NESM and the corresponding position of the platform are obtained.

We prepare the slope conditions by changing two orientation angles of the robot: the yaw angle around $z_{R}$ and the roll angle around $x_{R}$ in $\Sigma_{R}$. The roll angle represents the inclination of the slope. The yaw angle represents the front direction of the robot. We set the range of the yaw angle from $0[\mathrm{rad}]$ to $2 \pi[\mathrm{rad}]$ every $0.2 \pi[\mathrm{rad}]$, and the roll angle from $0[\mathrm{rad}]$ to $\frac{\pi}{4}[\mathrm{rad}]$ every $\frac{\pi}{40}[\mathrm{rad}]$. Then we obtain a maximum values map of NESM with respect to the yaw and roll angles.

\subsection{Results}

Figure 11 shows the maximum values map of NESM with respect to the yaw and roll angles. The largest maximum NESM is observed as 0.0328 when the yaw angle is $\pi[\mathrm{rad}]$ and the roll angle is $\frac{3 \pi}{40}[\mathrm{rad}]$. When the robot keeps the same direction as the yaw angle of $\pi[\mathrm{rad}]$ and the inclination of the slope changes, the smallest maximum NESM

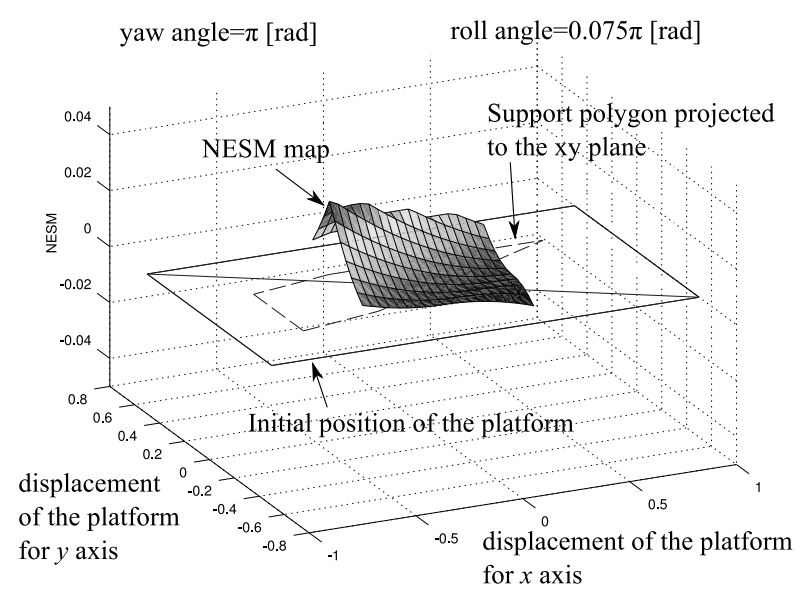

Figure 12. NESM map with respect to the $x-y$ displacement of the platform when the yaw angle is $\pi$ [rad] and the roll angle is $\frac{3 \pi}{40}[\mathrm{rad}]$ at the largest maximum NESM as shown in Figure 11.

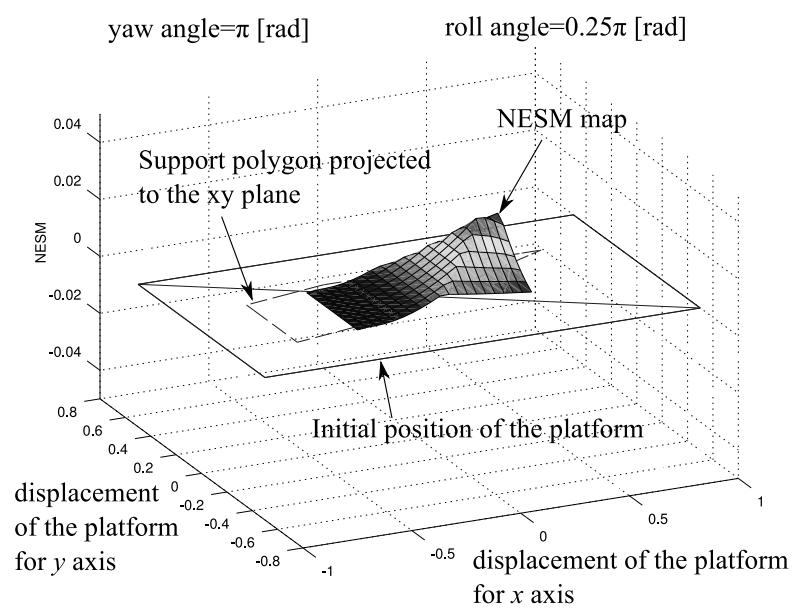

Figure 13. NESM map with respect to the $x-y$ displacement of the platform when the yaw angle is $\pi$ [rad] and the roll angle is $\frac{\pi}{4}[\mathrm{rad}]$. The robot keeps the same direction as Figure $12-$ i.e. the yaw angle of $\pi[\mathrm{rad}]$, where the maximum NEMS value is minimized at the roll angle of $\frac{\pi}{4}[\mathrm{rad}]$.

is observed as 0.0232 when the roll angle is $\frac{\pi}{4}[\mathrm{rad}]$.

Figure 12 shows the NESM map with respect to the $x-y$ displacement of the platform when the yaw angle is $\pi[\mathrm{rad}]$ and the roll angle is $\frac{3 \pi}{40}[\mathrm{rad}]$. Figure 13 is different from Figure 12 only in that the roll angle is $\frac{\pi}{4}[\mathrm{rad}]$. For the sake of comprehension, the projection of the support polygon to the $x y$ plane is drawn by a broken line. The solid rectangle represents the initial position of the platform. It is drawn on the zero-level plane of the NESM.

Figure 14 and Figure 15 show wire-frame models of the robot in the cases of maximum NESM in Figure 12 and Figure 13, respectively. Each of the platforms is painted gray. Under the platform, the gantry-shaped leg is represented by solid lines. The gripper positions of Legs 2, 3, and 4 form the support polygon designated by the broken lines.

Figure 16 shows the support polygons, the position of the COGs, and maximum height regarding the NESM re- 


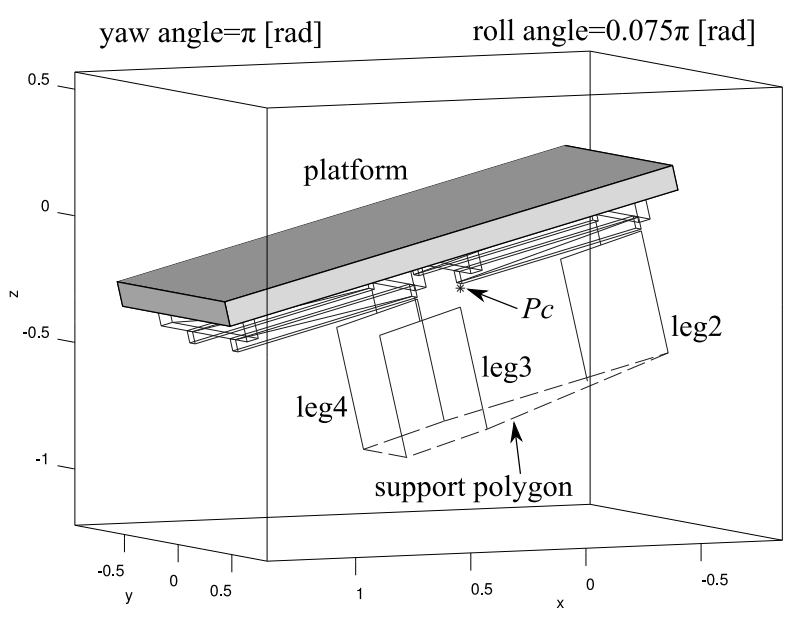

Figure 14. Relationship between COG and support polygon at the maximum NESM in the Figure 12. $p_{c}$ designates the position of COG.

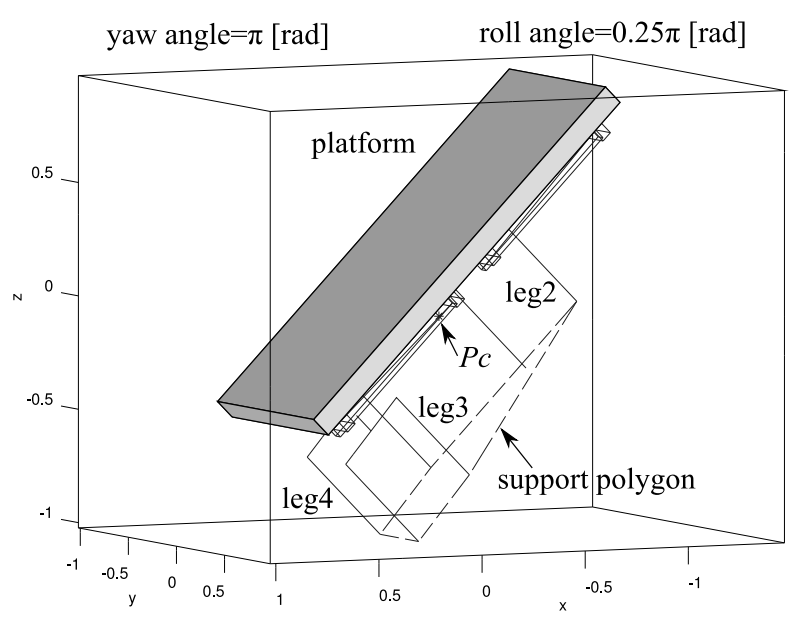

Figure 15. Relationship between COG and the support polygon at the maximum NESM in the Figure 13. $p_{c}$ designates the position of COG.

lationship in Figure 12 and Figure 13.

\section{Discussion}

We can observe in Figure 11 that the maximum NESM tends to decrease in general as the roll angle increases that is, the inclination of the slope becomes larger.

As shown in the preceding figures, the shape of the support polygon of the robot is long and narrow. It is easy to find that a stability margin against tumbling tends to be wider in the longer direction and narrower in the shorter direction. Figure 11 shows this tendency; that is, the maximum NESM is relatively high at the yaw angle around $0[\mathrm{rad}]$ or $\pi[\mathrm{rad}]$ and low around $\frac{\pi}{2}[\mathrm{rad}]$ or $\frac{3 \pi}{2}[\mathrm{rad}]$. Especially in the latter case, some of the maximum NESMs become negative when the roll angle is larger than $\frac{\pi}{8}[\mathrm{rad}]$.

Comparing Figure 12 and Figure 13, the position of the peek in the NESM map is different. According to Figure 16, the tip-over edge in the case of Figure 12 is a swing leg side on the support polygon. It likely happens in the

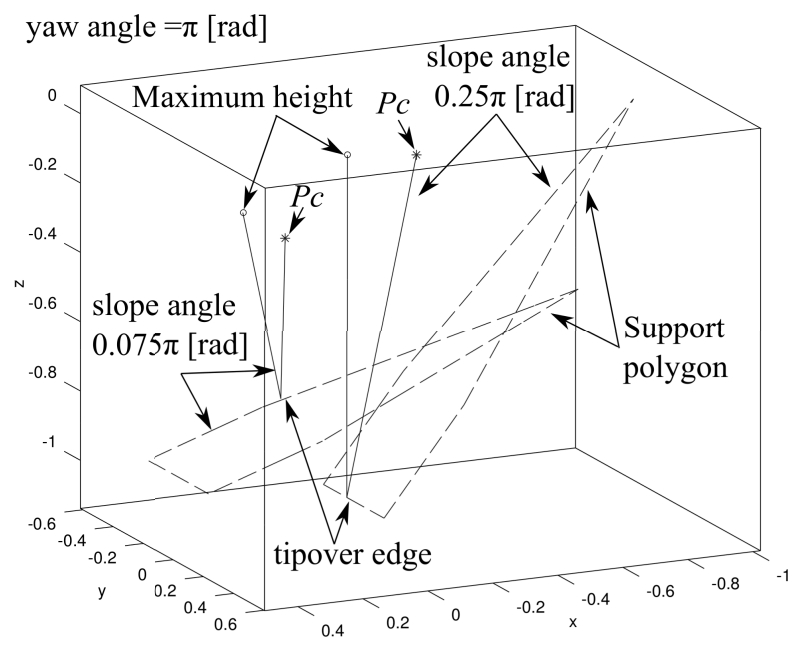

Figure 16. Relationship between COG and the support polygon when NESM is maximum and minimum. $p_{c}$ designates the position of COG.

swing phase of the walking motion on even terrain. On the other hand, the tip-over edge in the case of Figure 13 is a downhill side of the slope on the support polygon. This means that the tip-over edge of the support polygon depends on the steepness of the slope. To maintain a high enough NESM value during the walking motion, it is important to measure the steepness of the slope and check NESM at every moment.

In a real situation, the robot should move to avoid very steep terrain. The strength of a gantry-shape leg or the maximum force and torque of the actuators must not exceed the design specification. However, as shown in Figure 15 , very steep slope requires greater strength or force/torque. Theoretically, the positive value of NESM can maintain the robot's balance; however, too much inclination may damage the machine.

\section{Conclusions}

In this paper, we investigated the NESM values of a double-spiral mobile robot in the swing phase on several gradients of the slope from the numerical case study. We derived the support polygon from the kinematics analysis and showed the position vector of COG of the whole robot. Then, we described the way to obtain NESM values from the vector calculation.

The numerical case study revealed that the stability margin against tumbling tended to be wider in the longer direction of the body platform and narrower in the shorter direction. we reported the results and discussed the potential stability of the robot.

Even if the direction of the inclination of the slope was the same, if the degree of the inclination changed, the shape of the NESM map with respect to the position of the platform was also different. The double-spiral mobile robot was able to maintain its foot position while the position of the platform changed. Therefore, it is important to measure the steepness of the slope, to check NESM at ev- 
ery moment, and to move the platform in the appropriate direction to maintain a high enough NESM value in the walking motion.

The numerical case study discussed in this paper just showed the NESM map for a specific pose of the robot in a stationary condition. To investigate dynamic stability or to perform the dynamical simulation is left for future work.

\section{Acknowledgments}

This work was supported by JSPS KAKENHI Grant Number 24560282 and 16K06171. The authors also express their sincere gratitude to Center of Environmental Science and Disaster Mitigation for Advanced Research (CEDAR) at Muroran Institute of Technology for meaningful assistance.

\section{References}

C. B. Barber, D. P. Dobkin, and H. Huhdanpaa. The quickhull algorithm for convex hulls. ACM Trans. on Mathematical Software, 22(4):469-483, 1996.

H. Fujita, Y. Igarashi, S. Hotes, M. Takada, T. Inoue, and M. Kaneko. An inventory of the mires of Hokkaido, Japan - their development, classification, decline, and conservation. Plant Ecology, 200(1):9-36, 2009.

N. Hanajima, Y. Hayasaka, N. Azumi, K. Kawauchi, M. Yamashita, and H. Hikita. Double spiral propulsion mechanism in wetlands. In Joint Seminar on Environmental Science and Disaster Mitigation Research 2009, pages 77-78, 2009.

N. Hanajima, Q. Liu, and H. Kajiwara. A four-legged mobile robot with prismatic joints on spiral footholds. Technical Report 65, Memoirs of the Muroran Inst. of Tech., 2016.

S. Hirose, H. Tsukagoshi, and K. Yoneda. Normalized energy stability margin and its contour of walking vehicles on rough terrain. In Proc. IEEE Int. Conf. on Robotics and Automation (ICRA'2001), pages 181-186, 2001.

T. Kaneko and N. Hanajima. Evaluation of walking motion on the slope ground using normalized energy stability margin for a double spiral mobile robot. In Proc. 48th SICE Hokkaido Branch Conf., pages 31-32, 2016. (in Japanese).

R. B. McGhee and A. A. Frank. On the stability properties of quadruped creeping gaits. Mathematical Biosciences, 3(2): $331-351,1968$

D. A. Messuri and C. A. Klein. Automatic body regulation for maintaining stability of a legged vehicle during rough-terrain locomotion. IEEE Trans. Robot. Autom., RA-1(3):132-141, 1985.

H. Musgrave and A. Binley. Revealing the temporal dynamics of subsurface temperature in a wetland using time-lapse geophysics. Journal of Hydrology, 396(3-4):258-266, 2011.

F. Nakamura, S. Kameyama, and S. Mizugaki. Rapid shrinkage of kushiro mire, the largest mire in japan, due to increased sedimentation associated with land-use development in the catchmen. Catena, 55(2):213-229, 2004.
E. G. Papadopoulos and D. A. Rey. A new measure of tipover stability for mobile manipulators. In Proc. IEEE Int. Conf. on Robotics and Automation (ICRA'96), pages 3113-3116, 1996.

E. S. Riddell, S. A. Lorentz, and D. C. Kotze. A geophysical analysis of hydro-geomorphic controls within a headwater wetland in a granitic landscape, through eri and ip. Hydrology and Earth System Sciences, 14(8):1697-1713, 2010. 